\title{
Case-control study of the treatment of postaxial polysyndactyly of the foot: Comparison of surgical results after removal of the fifth or sixth toe
}

\author{
Soo Jin Woo, Byung Jun Kim, Sung Tack Kwon \\ Department of Plastic and Reconstructive Surgery, Seoul National University College of Medicine, Seoul, Korea
}

Background In postaxial polysyndactyly of the foot, the choice of which toe to excise is controversial. It is often treated by resection of the fifth toe to save the lateral neurovascular bundles of the sixth toe. However, the sixth toe is often short and laterally deviated, which may require wedge osteotomy, potentially shortening the phalanx and compromising circulation. This study outlines an individualized method to spare the length and axis of the fifth toe in polysyndactyly with a short and deviated sixth toe.

Methods We retrospectively analyzed 38 patients who underwent surgery between 2006 and 2019. The fifth toe was spared in 18 cases, and the sixth toe in 20 cases. The ratios of the forefoot width, angle difference, and toe length were compared between the affected and unaffected sides postoperatively. Complications and subjective judgments on cosmetic results were recorded and compared.

Results No significant between-group differences were observed for sex, age at surgery, or the follow-up period. The forefoot width ratio did not significantly differ between the groups. However, the angle difference and toe length ratios showed significantly better results in the fifth toe-spared group than in the sixth toe-spared group $(\mathrm{P}<0.05$ and $\mathrm{P}<0.01$, respectively). There were no cases of impaired circulation, and subjective evaluations revealed satisfactory results in the fifth toe-spared group.

Conclusions In cases with short and deviated sixth toes, sparing the fifth toe is an effective method of cosmetic treatment. The surgical results were satisfactory, with an improved appearance and no residual deformities.

Keywords Postaxial / Polysyndactyly / Polydactyly / Toe
Correspondence: Sung Tack Kwon Department of Plastic and Reconstructive Surgery, Seoul National University College of Medicine, 101 Daehak-ro, Jongno-gu, Seoul 03080, Korea

Tel: +82-2-760-3759

Fax: +82-2-745-5986

E-mail: stk59@snu.ac.kr

\section{INTRODUCTION}

Polydactyly of the foot is among the most common congenital anomalies of the foot. It is characterized by six toes, with or without duplication of the corresponding metatarsals or phalanges [1]. The manifestations of polydactyly of the foot may range from vestigial soft-tissue masses to an additional fully formed digit. Depending on the position of the extra toe, polydactyly of the foot can be classified as preaxial, central, or postaxial. Among these, the postaxial type is the most frequently encountered [2]. Watanabe et al. [3] proposed a dichotomization of these abnormalities based on their appearance on radiography 
as fifth or sixth ray duplications (with phalangeal or metatarsal bifurcation, respectively). When the condition involves syndactyly of the fourth and fifth toes, it is referred to as polysyndactyly, which is characterized by a dominant fifth toe and a hypoplastic sixth toe.

Simple excision of the extra toes does not yield satisfactory results due to the diverse and complicated shapes of polysyndactyly of the foot. The choice of which toe to excise has a significant effect on the postoperative outcome and cosmesis, and remains a controversial issue. The commonly described treatment for postaxial type polysyndactyly involves resection of the fifth toe to preserve the neurovascular bundles of the fourth and sixth toes $[4,5]$. However, the sixth toe is occasionally noticeably short of reaching the arcade of toes, with disfigurement of the toenail. The sixth toe also has a variable degree of lateral axis deviation, which requires wedge osteotomy of the proximal phalanx to achieve better alignment with the remaining toes. The traumatic process of wedge osteotomy can lead to shortening of the phalanx with circulatory compromise, and this procedure does not consistently yield satisfactory outcomes.

The literature contains insufficient information regarding the management of polysyndactyly of the foot. This study aimed to introduce an individualized method for treating patients with postaxial polysyndactyly of the foot by sparing the fifth toe and removing the short and deviated sixth toe. We also compared the surgical results to those of the previous method, which involved saving the sixth toe instead of the fifth, to verify the advantages of this individualized method.

\section{METHODS}

\section{Patients and data collection}

We retrospectively evaluated 38 patients with postaxial polysyndactyly of the foot with short and deviated sixth toes who underwent surgery by a single surgeon (STK) between September 2006 and July 2019. We compared 18 cases treated by sparing the fifth toe to 20 cases treated by sparing the sixth toe. Cases of polysyndactyly without syndactylism, bilateral cases, and syndromic cases were excluded from the study. The patients' demographic characteristics and clinical information were extracted from their medical records. The SAM system was used to classify the anomalies using preoperative X-rays and medical photographs [6]. This study received approval from the Institutional Review Board (IRB No. 2003-088-1109). All patients provided written informed consent prior to enrollment.

\section{Assessment}

Objective results were determined via anterior-posterior (A-P) foot X-rays. The forefoot width was determined preoperatively by measuring the distance between the widest points of soft-tissue margins in the transverse axis of the foot (Fig. 1, A). This point was typically at the level of the interphalangeal joint of the great toe and proximal interphalangeal (PIP) joint of the sixth toe. The orderly alignment of the toes' arcade was then evaluated by comparing the angle difference in the alignment of toes before and after surgery (Fig. 1, B). Two lines connecting the midpoints of the tips of the two outermost adjacent toes were drawn on A-P foot X-rays. The angle between the two lines was measured. Lastly, the fifth and sixth toe length before surgery and the fifth toe length after surgery were measured as the distance between the tip of the distal phalanx to the base of the proximal phalanx (Fig. 1, C). A postoperative evaluation was performed at the final follow-up session. In each patient, the affected side was compared to the unaffected side before and after surgery.

As the participants were too young to cooperate, their guardians subjectively evaluated the outcomes based on appearance and current symptoms using a similar method to that used by Phelps and Grogan [7]. Outcomes were evaluated in the following areas: symptoms, callosities, residual deformities such as valgus or varus deformities, and cosmetic appearance. A "satisfac-

\section{Fig. 1. Measurements on A-P foot X-rays}

Objective results were assessed using A-P foot $X$-rays preoperatively and postoperatively. A: Forefoot width was measured at the widest length of the foot horizontally. B: The angle difference was assessed by drawing two linear lines that connected the midpoint of the tips of the outermost two adjacent toes and measuring the angle between the two lines. C: Toe length was measured as the distance between the tips of the distal phalanx to the base of the proximal phalanx. A-P, anterior-posterior.

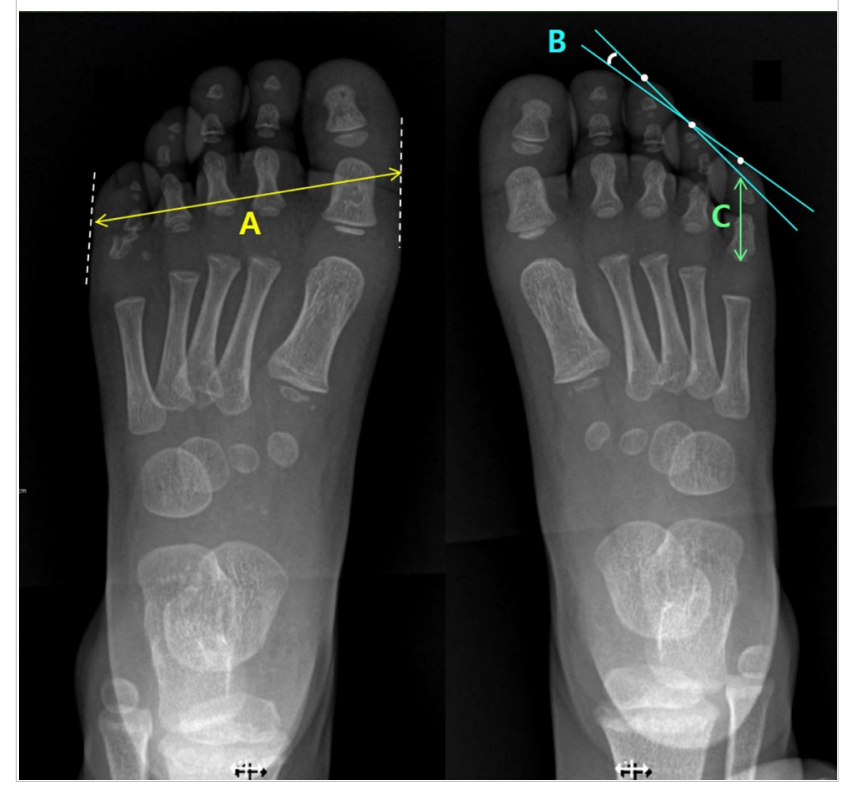


tory" rating was given to patients with no pain, callosities, or residual deformities who had a cosmetically acceptable appearance. If a suboptimal evaluation was given in any of the four categories, the patient's outcome was rated as "unsatisfactory."

\section{Surgical technique}

In the fifth toe-spared group, the fourth and fifth toes were separated via an interdigital zigzag incision, taking care not to disrupt the medial neurovascular bundle of the fifth toe (Fig. 2A). After confirmation that the neurovascular bundles of the fifth toe were intact, a second interdigital zigzag incision was made at the lateral side of the fifth toe to expose the phalangeal bones and metatarsal bone. The sixth toe was excised while preserving the medial portion of the head of the proximal phalangeal bone to prevent instability and stiffness of the PIP joint.

In the sixth toe-spared group, an interdigital incision was made between the fourth and fifth toes (Fig. 2B). After exposure of the phalangeal bones, the fifth toe was removed. If the sixth toe was laterally deviated, wedge osteotomy was performed at the proximal phalanx and fixated with two or three Kirschner wires. In both groups, the remaining skin defect between the fourth and fifth or sixth toe was covered using full-thickness skin harvested from the inguinal area to achieve tension-free wound closure. After application of an aseptic compressive dressing, a short leg splint was applied for 10 days.

\section{Statistical analysis}

Results are presented as the mean \pm standard deviation. The unpaired t-test, Pearson chi-square test, and Wilcoxon rank-sum test were used for between-group comparisons. The statistical analyses were conducted using PASW Statistics 18 (SPSS Inc., Chicago, IL, USA). P-values $<0.05$ were considered to indicate statistical significance.

\section{RESULTS}

The demographic information and clinical features of the patients are shown in Table 1. No significant differences were found between the two groups regarding sex distribution, the

Table 1. Demographic information and clinical characteristics of the patients

\begin{tabular}{lccc}
\hline Characteristics & $\begin{array}{c}\text { Fifth toe- } \\
\text { spared group }\end{array}$ & $\begin{array}{c}\text { Sixth toe- } \\
\text { spared group }\end{array}$ & P-value \\
\hline No. of patients & 18 & 20 & - \\
Sex (female/male) & $12 / 6$ & $9 / 11$ & 0.18 \\
Age at time of surgery (mon) & $17.8(10-68)$ & $16.1(9-31)$ & 0.39 \\
SAM classification & & & \\
S & & & - \\
0 & 0 & 0 & \\
1 & $13(72.2)$ & $13(65)$ & \\
2 & $5(27.8)$ & $7(35)$ & \\
A & & & \\
0 & $2(11.1)$ & $3(15)$ & \\
1 & $10(55.6)$ & $6(30)$ & \\
2 & $6(33.3)$ & $11(55)$ & \\
M & & & \\
0 & $18(100)$ & $20(100)$ & \\
1 & 0 & 0 & \\
2 & 0 & 0 & \\
Follow-up period (mon) & $20.5(6-38)$ & $23.4(9-61)$ & 0.38 \\
\hline
\end{tabular}

Values are presented as median (range) or number (\%).

S, syndactylism; A, axis deviation; $M$, metatarsal extension.

\section{Fig. 2. Schema of the surgical procedures}

The blue-colored area indicates the removed part. Neurovascular bundles are drawn at the lateral side of the fourth toe and medial side of the fifth toe. The red linear line indicates the osteotomy line. (A) Sparing the fifth toe. The fourth and fifth toes are separated with care not to injure the fifth toe's medial neurovascular bundle. When excising the sixth toe, the medial portion of the head of the proximal phalangeal bone is preserved. (B) Sparing the sixth toe. Wedge osteotomy of the sixth toe is performed to correct the lateral deviation of the toe.
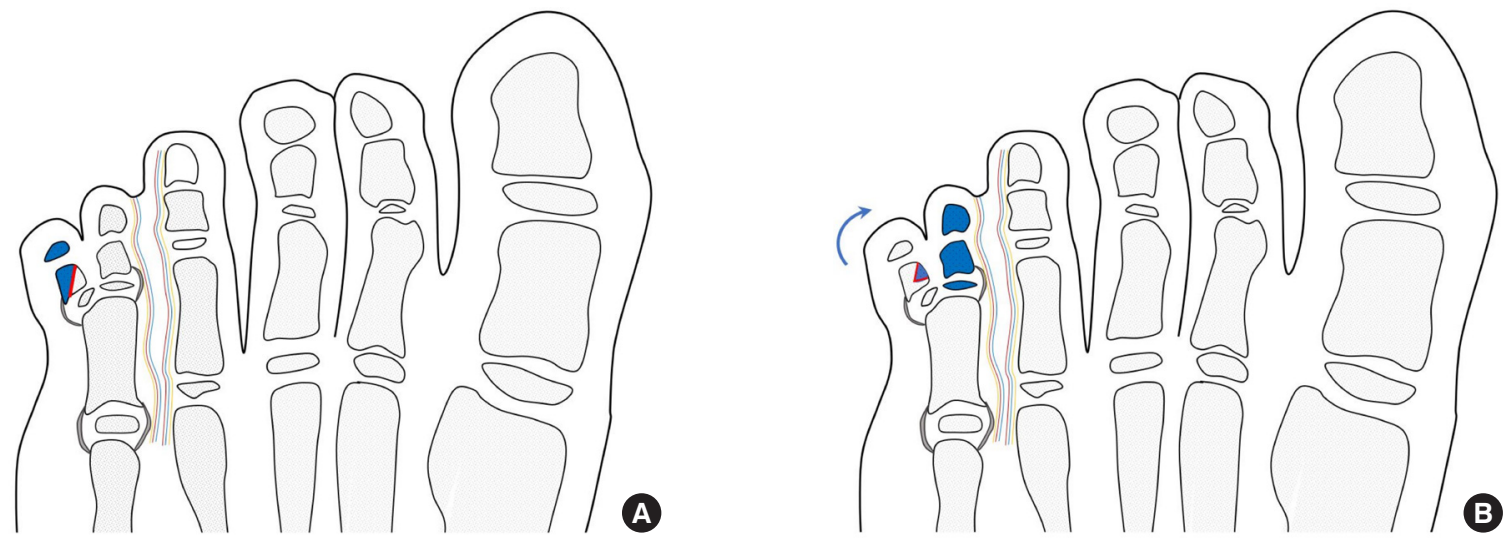
average age at the time of surgery, and follow-up duration. There were no S0-type cases in either group, given that all patients had some degree of syndactylism with the adjacent toe. All cases were $\mathrm{M} 0$ with no metatarsal involvement of the extra digit.

Table 2 shows the forefoot width, angle difference, and toe length before and after surgery in both groups. The postoperative ratio of the affected to unaffected side was calculated for each category. The closer the ratio was to 1 , the greater the similarity between the affected and unaffected sides. No significant difference was found between the two groups regarding the forefoot width ratio. However, the angle difference ratio significantly differed between fifth toe-spared group and sixth toespared group ( 1.23 vs. $1.59, \mathrm{P}<0.05$ ). The toe length ratio also significantly differed between the two groups ( 0.99 vs. 0.74 , $\mathrm{P}<0.01$ ).

Satisfaction with the results showed a significant difference between the two groups $(\mathrm{P}<0.05)$ (Table 3$)$. In the fifth toespared group, guardians were satisfied with the overall appearance of the foot and the spared toe, without residual deformity or lateral deviation (Figs. 3, 4).

There were no postoperative complications such as wound dehiscence, wound infection, bone growth delay, or bone malunion in either group until the final follow-up visit. However, in the fifth toe-spared group, there were two cases of hypertrophic scarring, which were not sufficiently severe to necessitate additional surgery, and one case of callus formation, which required reoperation. In the sixth toe-spared group, there was one case of hypertrophic scarring, one case that resulted in total loss of the

Table 3. Satisfaction of the patients' guardians after surgery

\begin{tabular}{lccc}
\hline Satisfaction & $\begin{array}{c}\text { Fifth toe- } \\
\text { spared group }\end{array}$ & $\begin{array}{c}\text { Sixth toe- } \\
\text { spared group }\end{array}$ & P-value \\
\hline Satisfactory & 15 & 10 & 0.036 \\
Unsatisfactory & 3 & 10 & \\
\hline
\end{tabular}

Outcomes were evaluated in four areas: symptoms, presence of callosities, residual deformities such as valgus or varus deformities, and cosmetic appearance. Satisfactory: feet with no pain, callosities, residual deformities, and cosmetically acceptable appearance; Unsatisfactory: feet with occasional pain, callosities, residual deformities, or an unacceptable scar appearance.

Table 2. Preoperative and postoperative measurements of forefoot width, angle difference, and toe length in both groups

\begin{tabular}{|c|c|c|c|c|c|c|c|}
\hline \multirow[b]{2}{*}{ Measurements } & \multicolumn{3}{|c|}{ Fifth toe-spared group } & \multicolumn{3}{|c|}{ Sixth toe-spared group } & \multirow[b]{2}{*}{ P-value } \\
\hline & Affected & Unaffected & $\begin{array}{c}\text { Affected/ } \\
\text { unaffected }\end{array}$ & Affected & Unaffected & $\begin{array}{c}\text { Affected/ } \\
\text { unaffected }\end{array}$ & \\
\hline \multicolumn{8}{|l|}{ Forefoot width (mm) } \\
\hline Preoperative & $59.23 \pm 4.18$ & $56.79 \pm 3.96$ & - & $58.53 \pm 3.89$ & $55.79 \pm 2.97$ & - & - \\
\hline Postoperative & $63.06 \pm 4.73$ & $62.48 \pm 4.65$ & $1.01 \pm 0.02$ & $62.28 \pm 3.74$ & $61.99 \pm 3.51$ & $1.00 \pm 0.02$ & 0.46 \\
\hline \multicolumn{8}{|l|}{ Angle difference $\left({ }^{\circ}\right)$} \\
\hline Preoperative & $15.51 \pm 2.87$ & $8.74 \pm 2.08$ & - & $18.78 \pm 3.31$ & $9.21 \pm 2.65$ & - & - \\
\hline Postoperative & $9.06 \pm 2.37$ & $8.32 \pm 3.33$ & $1.23 \pm 0.55$ & $14.74 \pm 4.22$ & $9.53 \pm 1.89$ & $1.59 \pm 0.52$ & 0.04 \\
\hline \multicolumn{8}{|l|}{ Toe length $(\mathrm{mm})$} \\
\hline Preoperative & $\begin{array}{l}16.59 \pm 2.62 \text { (5th) } \\
13.59 \pm 2.17 \text { (6th) }\end{array}$ & $16.57 \pm 1.97$ & - & $\begin{array}{l}16.25 \pm 2.10 \text { (5th) } \\
13.06 \pm 2.40 \text { (6th) }\end{array}$ & $16.84 \pm 2.20$ & - & - \\
\hline Postoperative & $19.91 \pm 2.85$ & $20.08 \pm 2.10$ & $0.99 \pm 0.07$ & $14.57 \pm 2.23$ & $19.91 \pm 2.14$ & $0.74 \pm 0.13$ & $<0.001$ \\
\hline
\end{tabular}

Values are presented as mean \pm SD.

\section{Fig. 3. Case 1 in the fifth toe-spared group}

(A) Preoperative photograph and X-ray of a 12-month-old male patient with right postaxial polysyndactyly, type S1A1M0. (B) Photograph and Xray 7 months after surgery. The fifth toe was spared. While discarding the sixth toe, a section of proximal phalangeal bone was saved to support the mid-phalanx of the fifth toe. The orderly arcade of the toes of the patient's right foot was well preserved, and the length of the new fifth toe was comparable to the unaffected side.
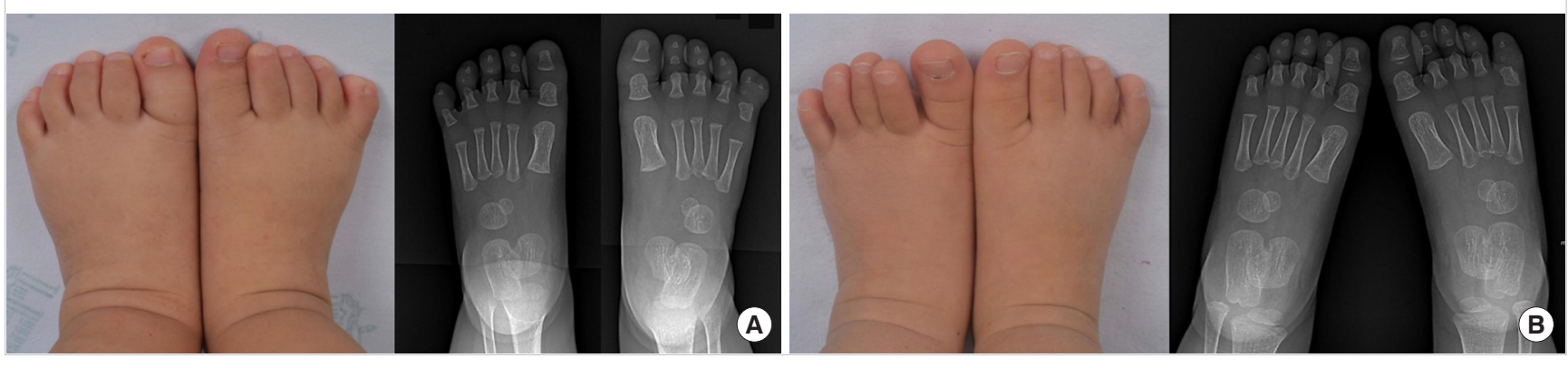


\section{Fig. 4. Case 2 in the fifth toe-spared group}

(A) Preoperative photograph and X-ray of a 14-month-old female patient with left postaxial polysyndactyly, type S2A1M0. (B) Postoperative photograph and X-ray after 30 months. The fifth toe was also spared in this case. The arcade of the left foot was well preserved, without a substantial angle difference from the contralateral foot.
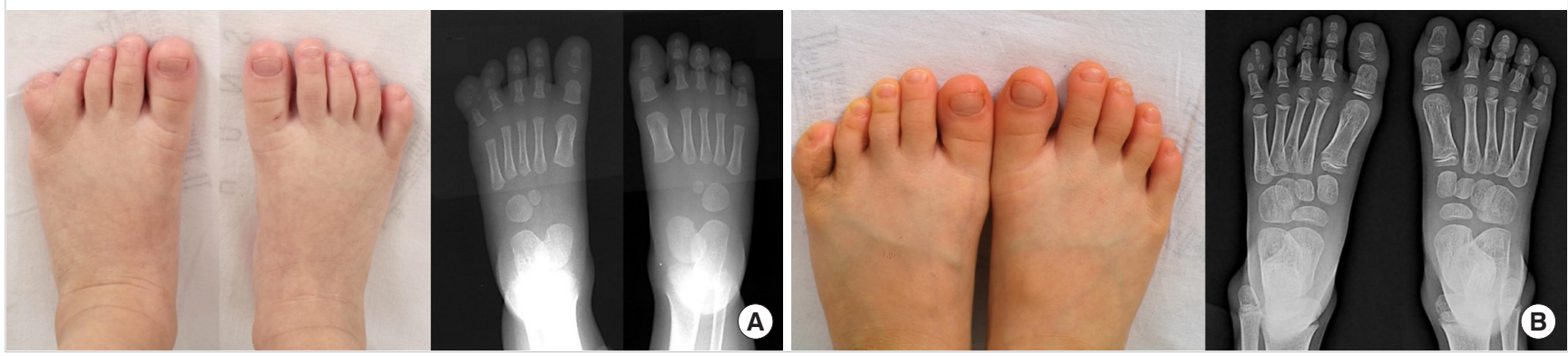

Fig. 5. Case in the sixth toe-spared group

(A) Preoperative photograph and X-ray of a 10-month-old male patient with left postaxial polysyndactyly, type S1A1M0. (B) Photograph and $\mathrm{X}$-ray 61 months after surgery. The sixth toe was spared, and wedge osteotomy was performed for the correction of lateral deviation. The toenail is missing, and the new fifth toe length is abnormally short of reaching the arcade of the rest of the toes due to circulatory compromise and procedure of wedge osteotomy.
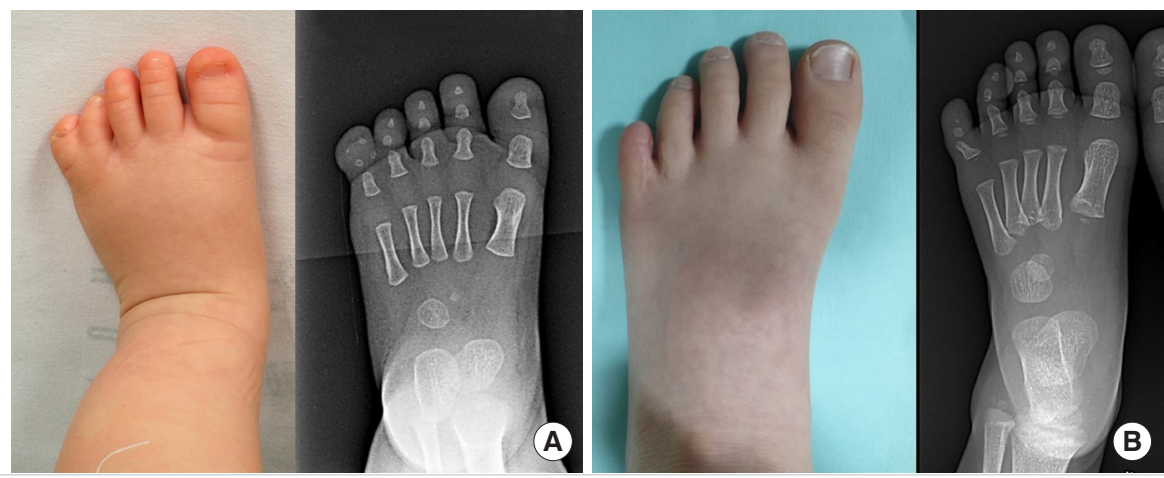

toenail (Fig. 5), and three cases of valgus deformity, of which one required reoperation of wedge osteotomy 2 years after initial surgery.

\section{DISCUSSION}

Polydactyly of the foot is among the most commonly encountered anomalies of the limbs, and accounts for $45 \%$ of congenital foot abnormalities [8-10]. This condition results from established genetic syndromes in some cases, but most frequently occurs as an isolated trait with an autosomal dominant pattern of inheritance and variable expressivity (gene penetrance) [11]. Polysyndactyly of the feet primarily affects the fifth toes, while polysyndactyly of the hand most commonly affects the third and fourth fingers of the hands, with partial or complete digital duplication within the affected web $[12,13]$. This condition involves a dominant mutation with variable expression in the HOXD13 gene, which encodes a transcription factor that plays an essential role in the development of the limbs [14]. Altered HOXD13 expression affects the embryonic development of the foot, resulting in polysyndactyly [12].

Numerous suggestions have been made regarding the classifi- cation of polydactyly of the foot. Conventional classifications are based on only morphology and do not reflect surgical planning or predict postoperative outcomes. In this study, the SAM system [6] was used for classification. This system can be used to customize surgical methods and preoperatively predict outcomes such as residual deformities. The category of syndactylism (S) reflects the requirement for skin grafts during surgery. In this study, all patients received skin grafts. The category of axis deviation (A) measures the axis deviation of the toe to be preserved and indicates the need for wedge osteotomy. The average axis deviation of the toe to be preserved was $21.3^{\circ}$ in the fifth toe-spared group and $30.5^{\circ}$ in the sixth toe-spared group. In the fifth toe-spared group, no cases required wedge osteotomy, whereas $85 \%$ of cases needed wedge osteotomy in the sixth toespared group. The category of metatarsal extension (M) describes the extent of metatarsal duplication. All of our cases were classified as M0, indicating no involvement of the metatarsal bone. Therefore, the primary purpose of reconstruction in this study was to achieve aesthetic improvement, rather than functional improvement.

Decisions regarding which toe to excise and the method by which this is achieved are regularly encountered clinical issues 
in polydactyly surgery because these aspects have a significant effect on the postoperative results and appearance $[7,15,16]$. Usami et al. [1] described four cases of on-top plasty to lengthen the toe in short-type postaxial polydactyly of the foot. The procedure involved separating and elevating the fifth toe with the neurovascular bundle in the metatarsophalangeal joint (distal component), followed by transferring the elevated toe ray to the top of the remaining proximal phalanx (proximal component) of the neighboring sixth toe after osteotomy. The results showed improvement in the total bone length and correction of the valgus deformity. Iba et al. [17] determined the toe to be excised depending on the appearance, alignment, and radiographic findings and focused on reconstructing the collateral ligament. The repair was performed with a proximally-based ligament or periosteal sleeve from the excised toe, and there were no cases of varus or valgus deformities.

An advantage of sparing the fifth toe is that excising the lateral sixth toe is an easier approach than excising the medial fifth toe. Furthermore, it is markedly less complicated than on-top plasty, but still yields highly satisfactory results. Although dividing the fourth web space and excising the sixth toe puts the fifth toe at risk of damaging the neurovascular bundles on both sides, there was no circulatory compromise, wound problems, or toe loss in this study. The preserved fifth toe exhibited no axis deviation and did not require wedge osteotomy, and the toe length was comparable to that of the unaffected side. Therefore, we recommend sparing the fifth toe if the sixth toe is too short or laterally deviated (A1 and $\mathrm{A} 2$ in the SAM classification).

In previous reviews, it was pointed out that excision of the lateral toe can lead to two common complications: valgus deformity and postoperative pain due to callus formation $[7,18,19]$. In this study, one case in the fifth toe-spared group experienced painful postoperative callus formation, which required removal 2 years after the primary surgery. However, there were no cases of valgus deformities. During the procedure of preserving the fifth toe, a small component of the proximal phalanx of the sixth toe was preserved to support the middle phalanx of the fifth toe. The disadvantage of this procedure is a relatively thick fifth toe after surgery due to the presence of two bones in the proximal phalanx. However, the thickness was not unpleasant enough to reduce cosmetic satisfaction or induce any discomfort while wearing shoes.

In this study, the angle difference was used as a measurement to reflect both the length and lateral deviation of the most lateral toe. This parameter indicated the degree of order in the arcade of the toes. If the most lateral toe is either very short or laterally deviated, the angle difference is larger, reflecting a disturbance of the orderly arcade of the toes. In sixth toe-spared group, the angle difference on the affected side did not always improve postoperatively. The preserved sixth toe was short and became shorter after wedge osteotomy, which led to a greater angle difference.

The limitations of this study include the relatively short followup period for confirming the postoperative results. The A-P foot $\mathrm{X}$-rays used for measurements were not consistently acquired vertically above the foot with full extension of all toes due to difficulties in the cooperation of the children. Additionally, the children's guardians assessed subjective satisfaction, instead of the children themselves. However, the results were compared between two similar demographic groups of patients. In each group, values were compared in ratios to overcome the varying rate of growth in children. This study also provided exact numbers for critical lengths and angles, which reflect aesthetic aspects of the foot. Nonetheless, further research is needed to assess the vascular structure of the fifth toe accurately. Subsequently, assessments of the tendon, ligament, and movement of the toe should be conducted using magnetic resonance imaging and ultrasonography to reinforce the final decision.

In cases of postaxial polysyndactyly in which simultaneous web reconstruction is required, the medial toe tends to be excised. However, in cases with short sixth toes with axis deviation, sparing the fifth toe reduces the need for wedge osteotomy, yielding a safe and effective treatment with high cosmetic satisfaction.

\section{NOTES}

\section{Conflict of interest}

No potential conflict of interest relevant to this article was reported.

\section{Ethical approval}

The study was approved by the Institutional Review Board of Seoul National University Hospital (IRB No. 2003-088-1109) and performed in accordance with the principles of the Declaration of Helsinki. Written informed consent was obtained.

\section{Patient consent}

The patient's guardian provided written informed consent for the publication and the use of the patient's images.

\section{Author contribution}

Conceptualization: ST Kwon. Data curation: SJ Woo. Formal analysis: SJ Woo. Methodology: BJ Kim, ST Kwon. Writing original draft: SJ Woo. Writing - review \& editing: SJ Woo, BJ Kim, ST Kwon. 


\section{ORCID}

Soo Jin Woo https://orcid.org/0000-0003-3476-8133

Byung Jun Kim https://orcid.org/0000-0002-6891-3768

Sung Tack Kwon

\section{REFERENCES}

1. Usami S, Kodaira S, Okazaki M. Primary on-top plasty for treatment of short-type postaxial polydactyly of the foot. Ann Plast Surg 2016;77:223-5.

2. Coppolelli BG, Ready JE, Awbrey BJ, et al. Polydactyly of the foot in adults: literature review and unusual case presentation with diagnostic and treatment recommendations. J Foot Surg 1991;30:12-8.

3. Watanabe H, Fujita S, Oka I. Polydactyly of the foot: an analysis of 265 cases and a morphological classification. Plast Reconstr Surg 1992;89:856-77.

4. Kojima T, Honma M, Sakurai T, et al. Postoperative evaluation of the polysyndactyly and its reparative techniques. Jpn J Plast Reconstr Surg 1972;15:336.

5. Aoki T, Nakajima T, Onishi K, et al. A new operative procedure using Z-plasty to repair polysyndactyly of the foot. Jpn J Plast Reconstr Surg 1991;34:833-7.

6. Seok HH, Park JU, Kwon ST. New classification of polydactyly of the foot on the basis of syndactylism, axis deviation, and metatarsal extent of extra digit. Arch Plast Surg 2013;40: 232-7.

7. Phelps DA, Grogan DP. Polydactyly of the foot. J Pediatr Orthop 1985;5:446-51.

8. Son WG, Gwon ST, Lee SW. Simple classification of foot

polydactyly based on the status of metatarsal bone and varus deformity.J Korean Soc Plast Reconstr Surg 2004;31:501-5.

9. Miura T, Nakamura R, Imamura T. Polydactyly of the hands and feet.J Hand Surg Am 1987;12:474-6.

10. Kim JH, Kim BJ, Kwon ST. Foot syndactyly: a clinical and demographic analysis. Arch Plast Surg 2016;43:559-63.

11. McClintic BS. Five generations of polydactylism. J Hered 1935;26:141-4.

12. Albrecht AN, Kornak U, Boddrich A, et al. A molecular pathogenesis for transcription factor associated poly-alanine tract expansions. Hum Mol Genet 2004;13:2351-9.

13. Malik S, Grzeschik KH. Synpolydactyly: clinical and molecular advances. Clin Genet 2008;73:113-20.

14. Kozin SH. Upper-extremity congenital anomalies. J Bone Joint Surg Am 2003;85:1564-76.

15. Nogami H. Polydactyly and polysyndactyly of the fifth toe. Clin Orthop Relat Res 1986;204:261-5.

16. Venn-Watson EA. Problems in polydactyly of the foot. Orthop Clin North Am 1976;7:909-27.

17. Iba K, Wada T, Kanaya K, et al. An individualized approach to surgical reconstruction for lateral polydactyly of the foot with an emphasis on collateral ligament reconstruction. Plast Reconstr Surg 2012;130:673e-680e.

18. Sahin O, Kuru I, Akgun RC, et al. Metatarsal transfer for the treatment of post-axial metatarsal-type foot synpolydactyly: a new technique that allows for comfortable shoe wearing. Bone Joint J 2013;95-B:929-34.

19. Uda H, Sugawara Y, Niu A, et al. Treatment of lateral ray polydactyly of the foot: focusing on the selection of the toe to be excised. Plast Reconstr Surg 2002;109:1581-91. 